\title{
Abnormal peripheral auditory asymmetry in schizophrenia
}

\author{
E Veuillet, N Georgieff, B Philibert, J Dalery, M Marie-Cardine, L Collet
}

UMR CNRS 5020, Neurosciences et Systèmes Sensoriels, Hôpital Edouard

Herriot, Pavillon U, 3 Place d'Arsonval, 69437 Lyon Cedex 03,

France

E Veuillet

B Philibert

L Collet

Institut des Sciences Cognitives, 8 Avenue Rockefeller, 69373 Lyon Cedex 08, France N Georgieff

CHS Le Vinatier, 95 bd Pinel, 69500 Bron, France

J Dalery

JE 1882, Université Claude Bernard, Lyon, France

J Dalery

M Marie-Cardine

CHS Le Vinatier, 95 bd Pinel, 69500 Bron, France

M Marie-Cardine

Correspondence to: Dr E Veuillet

evelyne.veuillet@chu-lyon.fr

Received 26 April 2000 and in revised form

19 September 2000

Accepted 28 September 2000

\begin{abstract}
Objective-Auditory processing difficulties have been reported in schizophrenia. This study explores peripheral auditory function in patients with schizophrenia in whom certain early disturbances of auditory message filtering have been found and may be associated with certain abnormalities which are particularly localised in the left temporal lobe.
\end{abstract}

Methods-Otoacoustic emissions, including click evoked and spontaneous emissions and measurements of functioning of the medial olivocochlear efferent system were obtained from 12 chronic schizophrenic patients and compared with normative data recorded from 12 normal controls.

Results-Otoacoustic emission amplitudes and medial olivocochlear functioning were similar between the normal controls and schizophrenic patients; the schizophrenic patients did, however, differ from the normal controls in otoacoustic emission intensity and in medial olivocochlear asymmetry. A tendency to a higher number of spontaneous peaks, and a significantly higher click evoked otoacoustic emission response amplitude were found in the right ear compared with the left ear of schizophrenic patients. For the medial olivocochlear system, whereas normal controls showed greater attenuation in the right than in the left ear, schizophrenic patients lacked such an asymmetry.

Conclusion-In the absence of any attention task, the findings show disturbed peripheral lateralisation in schizophrenia of mechanisms involved in auditory information filtering. Such a lack of right ear advantage in medial olivocochlear functioning may thus be a peripheral reflection of central lateralisation anomalies.

(F Neurol Neurosurg Psychiatry 2001;70:88-94)

Keywords: schizophrenia; auditory processing; asymmetry; medial olivocochlear efferent system; otoacoustic emissions; lateralisation

Schizophrenia is a clinical syndrome highly characterised by cognitive dysfunctions. But severe auditory perception deficiencies consistent with hypersensitivity to external stimuli are also reported. ${ }^{1}$ Deficient auditory processing has been widely reported in schizophrenia and such defects clearly appeared in auditory evoked responses. Schizophrenic patients show attenuation of the scalp recorded P300 re- sponse, ${ }^{23}$ the greatest difference with normal controls occurring at left temporal electrode sites. $^{45}$ Another electrophysiological abnormality is that the P50 component is less reduced in response to repeated stimulation, suggesting a deficit in the control of sensitivity to auditory stimuli. ${ }^{6-8}$ Abnormal mismatch negativity generation has also been reported in schizophrenic patients, ${ }^{9-11}$ particularly in the left hemisphere. ${ }^{12}$ This is consistent with a deficit in early cortical mechanisms of auditory sensory analysis. Finally, the auditory brainstem responses occurring within $10 \mathrm{~ms}$ of stimulation can also be affected in schizophrenia, with missing peaks or prolonged latencies. ${ }^{13}$ All in all, it seems that the early stages of auditory processing and perception are not intact in schizophrenic patients and that the underlying dysfunction may be an inability to properly filter the inflow of information from the environment. ${ }^{14}{ }^{15}$ This could lead to a decrease in ability to focus on external sensory stimuli and to the hyperawareness of background noise described in schizophrenic patients. ${ }^{16}$ Although electrophysiological studies tend to point to a disruption of initial encoding due to defective inhibitory mechanisms, the existence of abnormally reduced neuronal sensitivity leading to abnormal thresholds for novel stimuli can also be proposed. Few studies have looked for deficits in auditory performance in schizophrenia. Experiments using tonal audiometric procedures have shown that these abnormalities in auditory function appear in a context of normal auditory acuity, except that schizophrenic patients do not consistently show the usual auditory threshold asymmetry in favour of the right ear. ${ }^{17}$ In a more recent study, Mathew et al found a degradation in hearing thresholds in schizophrenic patients, with better right ear acuity only in the non-hallucinating patients. ${ }^{18}$ These data point to a loss of threshold asymmetry in one (hallucinating) subgroup of schizophrenic patients.

There is thus evidence from many sources that auditory input integration and perception are disturbed in schizophrenia. In auditory information processing, the signal undergoes encoding and initial filtering as early as the cochlea. In 1992, Dallos showed that the outer hair cells of the organ of Corti have a primordial role in encoding the signal which is to be received and processed by the inner hair cells and then transmitted by auditory nerve afferent neurons. ${ }^{19}$ These outer hair cells, in turn, are the target of medial olivocochlear neurons ${ }^{20}$ originating in the superior olivary complex. $^{21} 22$ It has been shown in animals that electrical or acoustic stimulation of medial 
olivocochlear fibres alters the nervous message released in the afferent auditory fibre (see Guinan $^{23}$ for a review). There is increasing evidence that the efferent fibres of the olivocochlear bundle, by innervating the outer hair cells, can affect sensory processing at the periphery. In humans, this medial olivocochlear system can be easily explored by means of otoacoustic emissions, which are sounds of cochlear origin..$^{24}$ It is now generally accepted that the origin of otoacoustic emissions lies in the outer hair cells, which undergo active mechanical displacement through the action of the contractile elements, myosin and actin, contained in their infrastructure. ${ }^{25}{ }^{26}$ In response to sound input, the outer hair cells change length, which causes cochlear vibration and which may in turn generate sound. A small fraction of this biological energy is retransmitted backwards in the form of a pressure wave to the tympanum via the oval window and ossicular chain. The consequent vibration of the tympanum generates a low level acoustic signal which can be detected with a sensitive microphone in the outer auditory canal. ${ }^{27}$ There are different types of otoacoustic emission: the first major class consists of continuous narrow band signals around one or more frequencies which can be found in the absence of any deliberate acoustic stimulation. They are known as "spontaneous otoacoustic emissions". Otoacoustic emissions can also occur as a broad band acoustic signal in response to an evoking click, and are then called "click evoked otoacoustic emissions. ${ }^{28}$ In humans, otoacoustic emissions are a unique tool for examining the cochlea, allowing direct non-invasive communication with the sensory cells. They are particularly sensitive in detecting hearing loss and the fact that they are absent in patients with hearing loss exceeding 25-30 dB HL (see Probst $e t a l^{28}$ for a review) shows that otoacoustic emission generation is related to optimal functioning of the ear. Kemp ${ }^{29}$ hypothesised that discontinuities in outer hair cell distribution modified the sound energy produced by otoacoustic emissions. Moreover, click evoked otoacoustic emissions have been found to decrease in amplitude when a noise is applied in the contralateral ear. ${ }^{30}{ }^{31}$ Such an effect, in which an efferent pathway is able to affect cochlear mechanics, was initially described in animal experiments. ${ }^{32} 33$ Evidence for efferent involvement comes not only from animal, ${ }^{34} 35$ but also from human studies, in which it has been reported that this contralateral suppression of click evoked otoacoustic emission is absent or greatly reduced in patients with vestibular neurectomy. ${ }^{36-38}$ As this section concerns the efferent fibres, these findings provide one of the main arguments for medial olivocochlear system involvement in the contralateral suppression effect. The medial olivocochlear system is part of the regulatory complex which modulates or regulates cochlear activity and auditory afferent inputs, improving the detection of multitone complexes in noise..$^{39-41}$ Abnormally reduced functioning of this system has been found in hyperacusia, ${ }^{42}$ and autism, ${ }^{43}$ two diseases in which impaired auditory processing is suspected, as is also the case in schizophrenia. Moreover, anatomical animal studies have shown neurophysiological connections between certain cortical areas and the superior olivary complex, ${ }^{44}$ which raise the possibility of central control of this modulatory olivocochlear feedback. Recent studies have shown that the medial olivocochlear system shows a pattern of functional asymmetry which is influenced by handedness, ${ }^{45}{ }^{46}$ and so could be the reflection of a central laterality.

These peripheral auditory mechanisms and their regulation by the CNS have never been studied in schizophrenia. The aim of the present study was therefore to compare cochlear mechanics and their central efferent suppression between schizophrenic patients and normal control subjects, with objective and non-invasive methods. As auditory abnormalities are reported to be more consistent in hallucinating than in non- hallucinating schizophrenic patients, we included only patients who reported auditory hallucinations.

\section{Methods}

SUBJECTS

Twenty four subjects were recruited for the study. All gave written consent. Exclusion criteria included history of medical or neurological illness or trauma. Twelve were schizophrenic patients (four outpatients and eight inpatients) who met DSM-IV diagnostic criteria $^{47}$ for chronic schizophrenia (duration of illness $>2$ years) with a mean illness duration of 11.7 (SE 3.5) years (range 2-37 years) and a mean average age at symptom onset of 20.2 (SE 0.6) years (range 17-25 years). The clinical severity of the patients' illness was assessed by the brief psychiatric rating scale (BPRS), ${ }^{48}$ the scale for assessment of negative symptoms (SANS) ${ }^{49}$ and the scale for assessment of positive symptoms (SAPS). ${ }^{50}$ There were five paranoid, two disorganised, and five undifferentiated schizophrenic patients, all reporting auditory hallucinations (score 4.0 (SE 0.4); range 2-6 years). At the time of testing, 10 patients were taking neuroleptic medication (mean daily medication dose in chlorpromazine-equivalents: 624 (SE 62.2) mg; range 380-950). Two patients had been free of any treatment for at least 4 weeks. Seven patients were also receiving antiparkinsonism treatment (tropatepine) with a mean dose of $14.3 \mathrm{mg}$ (SE 2.0); range 10-20). All patients were clinically stable at the time of evaluation and testing. The 12 other subjects were normal controls with no history of psychiatric illness or neurological disorder. The mean ages of the two groups were statistically similar (31.9 (SE 3.6) (range 21-58 years) for the schizophrenic patients, and 29.5 (SE 3.3) (range 18-57 years) for the normal controls (Mann-Whitney $U$ test $t=125.5, \mathrm{p}=0.712)$ ). Eight men and four women were included in each group. On the Edinburgh handedness scale, ${ }^{51}$ all subjects were right handed with scores above 70. Mean scores were statistically comparable between groups (92.5\% (SE 3.3); range 70-100) for the normal controls and $90.8 \%$ (SE 2.8); range 80-100) for the schizophrenic patients. 
HEARING TESTS

The battery of four tests was typically administered in the following order:

Pure tone audiogram

Thresholds at 250, 500, 1000, 2000, 4000, and $8000 \mathrm{~Hz}$ were measured in a soundproof room using a Madsen DAIII audiometer. All the subjects included in this study had auditory threshold losses of less than $25 \mathrm{~dB} \mathrm{HL}$ at all frequencies.

Tympanogram

Standard admittance screening was conducted using a manual tympanometer (Amplaid 702) to check the functional integrity of the middle ear. The threshold of the acoustic reflex to contralateral broadband noise was also measured. All the subjects included in the present study (normal controls and schizophrenic patients) had a stapedial reflex with threshold never below $80 \mathrm{~dB}$ SPL or above 100 $\mathrm{dB}$ SPL. The mean values obtained in the right and left ears did not differ significantly between the two groups.

Otoacoustic emission recordings and medial olivocochlear exploration

All the measures were made in a soundattenuated chamber, using the ILO92 equipment (Otodynamics ${ }^{\circledR}$ software, version 3.94 L), described in detail elsewhere. ${ }^{52}$ The right ear and left ear were investigated in random order. The subjects were instructed to relax, lie quietly, and remain awake. A probe was sealed with a perforated foam tip in the outer ear canal. Click evoked otoacoustic emissions were obtained in response to a non-filtered click of $80 \mu$ s duration, presented at a repetition rate of $50 / \mathrm{s}$. Three hundred responses were averaged with a band pass of $500-6000 \mathrm{~Hz}$. A first recording used the non-linear differential mode to obtain click evoked otoacoustic emission at a high stimulus intensity of around 83 (SE 3) dB SPL (sound pressure level) for a time window of between 2.6 and 20 ms. Spontaneous otoacoustic emissions were then looked for. Finally, the medial olivocochlear system was explored by recording click evoked otoacoustic emissions with and without contralateral acoustic stimulation, following a protocol described elsewhere. ${ }^{42}$ Click evoked otoacoustic emission recording conditions were the same as above, except that five stimulus intensities, ranging between 60 and $72 \mathrm{~dB}$ SPL in $3 \mathrm{~dB}$ steps, were applied in random order using the linear mode. For each intensity, one recording with and one recording without contralateral acoustic stimulation were successively made, in random order. Each response was windowed, blanking out the first $3.20 \mathrm{~ms}$. The contralateral acoustic stimulation was a $30 \mathrm{~dB}$ SPL continuous broad band noise (band width $50-8000 \mathrm{~Hz}$ ).

DATA PROCESSING

For each click evoked otoacoustic emission, a measure of total level ("echo") was given in $\mathrm{dB}$ SPL. In spontaneous otoacoustic emission recordings, the presence or absence of a spectrum peak was noted. A peak was retained if the signal to noise ratio exceeded $4 \mathrm{~dB}$. The number of peaks/ear was also noted. For medial olivocochlear exploration, the procedure allowed the equivalent attenuation to be calculated. The equivalent attenuation allows the quantification of medial olivocochlear functioning, and it is defined as the decrease in the ipsilateral stimulus $(\mathrm{dB})$ which causes the same reduction in click evoked otoacoustic emission amplitude as does a $30 \mathrm{~dB}$ SL contralateral acoustic stimulation. ${ }^{42} 53$ This calculation is made from the "echo" level presented at five intensities with and without contralateral acoustic stimulation (see above). The lateralisation of this contralateral suppression effect was quantified with an asymmetry index corresponding to the relative difference between equivalent attenuation for the right ear and equivalent attenuation for the left ear. A positive value indicates a better functioning of the medial olivocochlear system in the right ear and a negative value indicates a better functioning in the left ear.

\section{STATISTICAL ANALYSIS}

Analysis was performed with SigmaStat ${ }^{\circledR}$ software (Jandel Scientific). All data are expressed as mean (SE). The group effect, the ear effect, and the interaction of group and ear on total click evoked otoacoustic emission amplitude and equivalent attenuation were analyzed with two way repeated measures analysis of variance (RM ANOVA) on one factor. When the RM ANOVA was found to be significant at $p<0.05$ (for either the two factors or the interaction), a multiple comparison procedure was performed (Tukey test) to test differences between groups. A $t$ test was used to compare the asymmetry index between the two groups. $\mathrm{Z}$ Tests were used to compare proportions.

\section{Results}

OTOACOUSTIC EMISSIONS

Figure 1 A plots the mean values of click evoked otoacoustic emission response level (by group and ear). The two way RM ANOVA on click evoked otoacoustic emission total amplitude disclosed no effect of group but an ear effect $\left(F_{1,22}=9.85 ; \mathrm{p}=0.005\right)$. Post hoc analyses by Tukey test indicated that the click evoked otoacoustic emission amplitudes were significantly larger in the right ear than in the left ear $(p<0.005)$. The interaction effect between (group $\times$ ear) was significant $\quad\left(F_{1,22}=5.63\right.$; $\mathrm{p}=0.027)$. The post hoc Tukey test indicated that the difference between right ear and left ear reached significance only within the schizophrenic patient group $(\mathrm{p}<0.001)$.

For spontaneous otoacoustic emissions in the normal control group, the percentage of subjects with right ear spontaneous otoacoustic emissions was comparable with that for left ear spontaneous otoacoustic emissions (50\%). These percentages were not significantly different from those obtained for the schizophrenic patient group (58\% in the right ear and 33\% in the left ear). On the other hand, schizophrenic patients tended to present multiple spontaneous otoacoustic emissions more often than did normal controls (fig $1 \mathrm{~B}$ ). Although spontane- 

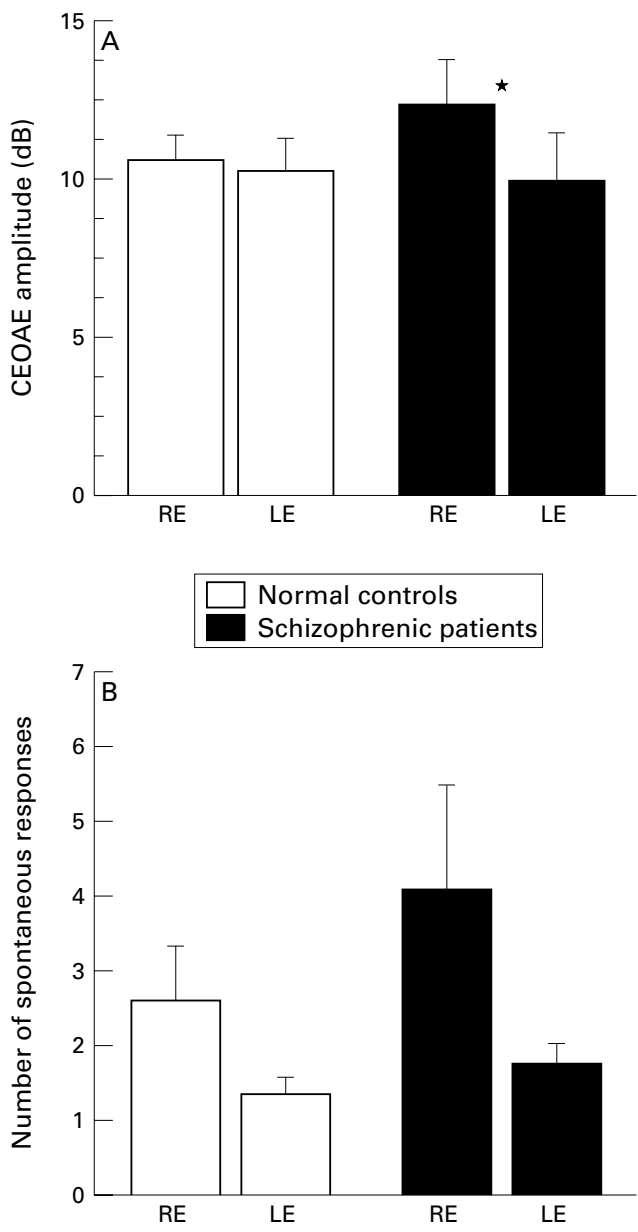

Figure 1 (A) Mean (SE) amplitude of click evoked otoacoustic emissions and (B) peak number of spontaneous otoacoustic emissions for the normal control subjects and schizophrenic patients. ${ }^{\star} p<0.05$.

ous otoacoustic emission peaks predominated in the right ear in both groups, the schizophrenic patients' right ear values tended to be higher. However, none of these differences reached significance.

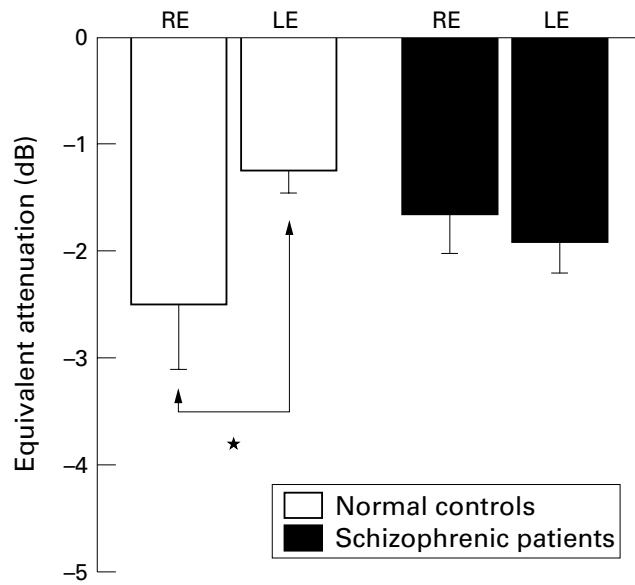

Figure 2 Mean (SE) equivalent attenuation obtained in normal control subjects and schizophrenic patients for the right and left ears. ${ }^{\star} p<0.05$. The more negative the equivalent attenuation, the greater the contralateral suppression of click evoked otoacoustic emissions.
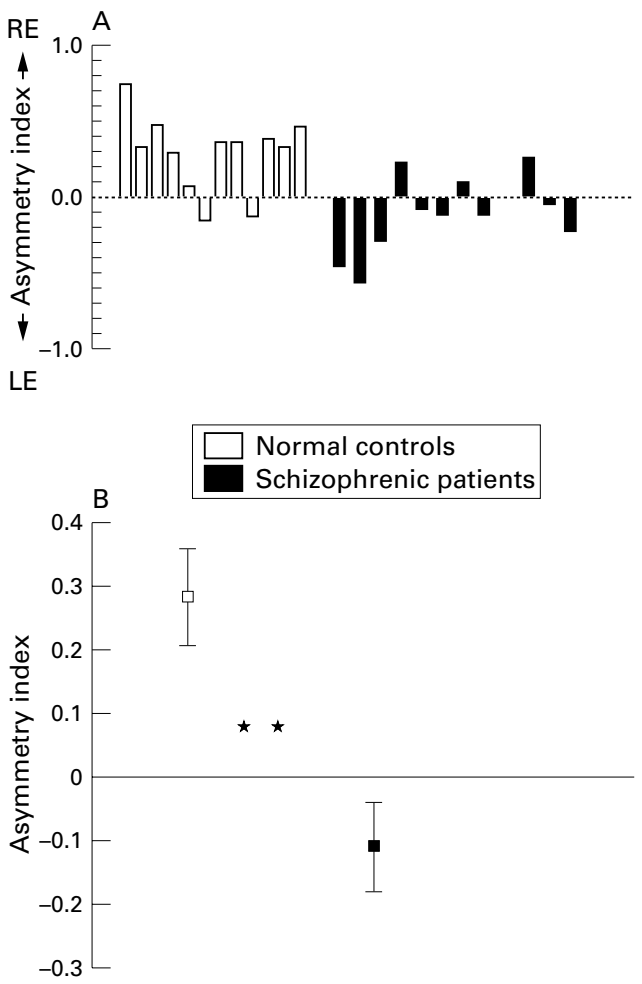

Figure 3 (A) Individual and (B) mean (SE) equivalent attenuation asymmetry index plotted according to group: normal controls and schizophrenic patients. A positive value indicates a right ear advantage and a negative value a left ear advantage. ${ }^{\star *} p<0.01$.

MOC MEASUREMENTS

Functioning

Figure 2 shows the mean absolute equivalent attenuation values for each group and ear. The contralateral suppressive effect was statistically comparable between groups, with a nonsignificant effect of group disclosed by two way RM ANOVA. There was, however, a significant effect of ear $\left(F_{1,22}=4.23, \mathrm{p}=0.05\right)$ and a significant interaction between group and ear $\left(F_{1}\right.$, $\left.{ }_{22}=10.13, p=0.004\right)$. Post hoc Tukey tests indicated that the normal controls showed a significantly greater contralateral suppressive effect in the right than the left ear $(p<0.001)$, and such asymmetry was not found in the schizophrenic patient group.

\section{Laterality}

This absence of systematic lateral difference in schizophrenic patients, unlike normal controls, is illustrated in fig 3. When the individual data are considered (fig $3 \mathrm{~A}$ ), it is seen that 10 out of 12 normal controls (83\%) presented significantly greater right ear suppression - that is, a right ear advantage - whereas of schizophrenic patients only three $(25 \%)$ showed such right ear advantage, eight presenting greater left ear suppression and one showing no laterality.

Mean asymmetry index values are plotted for both groups in fig $3 \mathrm{~A}$. They were significantly different between the two groups ( $t$ test: $t=3.32 ; \mathrm{p}<0.003$ ) but only the mean value of the normal control group differed from $0(t=3.93 ; \mathrm{p}<0.001)$. 


\section{Discussion}

This study is the first direct objective comparison between normal controls and schizophrenic patients of functional peripheral auditory processing thought to be involved in the filtering of acoustic input. As expected, schizophrenic patients differed from normal controls, but the distinction was found only in comparisons related to laterality: in all other cases, no significant difference appeared between groups.

Schizophrenic patients had otoacoustic emission amplitudes comparable with those of the normal controls, with however, particularly high click evoked intensity and spontaneous otoacoustic emission number in the right ear. The literature mentions fluctuating but significant rises in hearing threshold, particularly in hallucinating schizophrenic patients, in whom the asymmetry in favour of the right ear is absent. ${ }^{18}$ As otoacoustic emissions test inner ear functional integrity, and degrade rapidly when hearing thresholds rise, there would seem to be a mismatch between our data, obtained with an objective test, and the findings of Mathew et $a l,{ }^{18}$ obtained with a subjective test of hearing perception levels. There is, however, another possible interpretation. The presence of otoacoustic emissions is a reliable indicator of outer hair cell structural integrity. It can be hypothesised that higher click evoked otoacoustic emission amplitude and spontaneous otoacoustic emissions having multiple spectral peaks reflect an increase in cochlear amplification. Such a modification could affect nervous activity generated in the afferent, as found in animals, ${ }^{54}$ disrupting the processing of information, and which could perhaps explain the degradation of hearing threshold described. ${ }^{18}$ Medial olivocochlear functioning was quantitatively similar between normal controls and schizophrenic patients, suggesting that the auditory dysfunction found in such patients is different from the impairment described in autistic children, in whom the medial olivocochlear system was found to be significantly less functional than in controls. ${ }^{43}$ In fact, the main finding of the present study is that schizophrenic patients show an absence of normal functional medial olivocochlear asymmetry. Whereas mean medial olivocochlear functioning was better in the right ear of normal controls, confirming previous investigations, ${ }^{45}{ }^{46}$ right handed schizophrenic patients showed the kind of non-lateralised medial olivocochlear functioning seen in left handed normal controls. ${ }^{46}$ This absence of medial olivocochlear function asymmetry cannot be linked to the increased click evoked otoacoustic emission intensity in the right ear. ${ }^{55}{ }^{56}$ It is now well established that the auditory pathways are crossed, so that acoustic signals perceived in the right ear are integrated by auditory areas located in the left temporal cortex. Moreover, the planum temporale, thought to play an important part in language, is larger in this left hemisphere for normal right handed subjects, ${ }^{5758}$ receives direct auditory projections from the medial geniculate body - the subcortical relay nucleus for the auditory afferent system - and is probably involved in the early auditory processing. ${ }^{59}$ There is strong evidence that schizophrenic patients, particularly those with auditory hallucinations, have reduced cerebral asymmetry in the region of the planum temporale. (see Ross and Pearlson ${ }^{60}$ for a review ${ }^{61}$ ) Thus, the absence of functional medial olivocochlear asymmetry found in schizophrenic patients may be attributed to an impairment of the central modulation influencing the auditory efferent pathways, and so reflects the reduced central auditory asymmetry in these cortical areas. Based on this hypothesis, the present study could support the notion that schizophrenia involves certain abnormalities in cerebral laterality. ${ }^{62-64}$ However, no significant difference in suppression was found between normal controls and schizophrenic patients and it was not possible here to determine on which side (right or left) the medial olivocochlear system was more impaired and on which it was more activated.

Atypical lateralisation of auditory function in schizophrenic patients compared with normal controls has also been reported for other functional measures such as right ear advantage in dichotic listening tests, ${ }^{65} 66$ or $\mathrm{P} 300,{ }^{67}$ which, being subjective procedures requiring active participation from subjects, are thought to involve attention processes which are greatly impaired in schizophrenia. ${ }^{68}$ The strength of the present study is that all the procedures were objective, and subjects had no response to provide nor any cognitive task to perform. Our findings thus cannot be attributed to any alteration of attention processes, but rather identify, in a significant fraction of schizophrenic patients, disturbances indicating processing anomalies of auditory message perception as of peripheral level, which could in turn be related to anomalies in central lateralisation.

It is important to acknowledge several limitations of the present study. Firstly, there is the possible influence of the antipsychotic medication taken by most of the schizophrenic patients in this study. However, the primary effect of antipsychotic medication is an improvement in cognitive functions such as attention (see Green and King ${ }^{69}$ for a review. This effect has been seen to be lateralised, advantaging left hemisphere attentional processes (seeGruzelier ${ }^{70}$ for a review). The present study, however, used a methodology which does not rely on subjects' conscious processing, thus limiting the influence of cognitive processing and of the attention component in particular. Moreover, our findings with patients under neuroleptic drugs tend to show an absence of lateralisation, which seems to further confirm the fact that this procedure does not involve such attentional processes. Hence, there is no particular evidence that the absence of right ear advantage in medial olivocochlear functioning could be attributable to any such medication effect. On the other hand, this persistent loss of asymmetry even under medication might constitute a state independent vulnerability marker, as it seems to be the case for the P300 abnormalities found in the auditory mode. 
This is also an important argument against any significant impact of the second possible limitation of the present work, the existence of auditory hallucinations at test time, this factor being always difficult to quantify. For technical reasons, it must be underlined that the patients were always tested outside hallucinating periods.

Lastly, our study concerned peripheral auditory mechanisms only in schizophrenic patients who reported several times auditory hallucinations as compared with normal controls, and so does not provide data as to whether the abnormal peripheral auditory asymmetry in schizophrenia concerns psychosis in general, or is specific to schizophrenia itself, or indeed to auditory hallucinations. Further studies comparing subjects with psychotic depression, non-psychotic depression, and schizophrenia with and without auditory hallucinations seem to be warranted.

To summarise the present study is, to our knowledge, the first to explore objectively, in schizophrenia, certain functional auditory mechanisms thought to be under cerebral control, and provides a promising approach for the objective study of alterations in auditory perception in schizophrenia. This medial olivocochlear system lateralisation, which could be of importance in peripheral auditory filtering, could be an interesting "trait-like" cognitive marker in schizophrenia and its measurement might be usefully integrated in the functional exploration test battery, so as better to understand the auditory abnormalities which characterise some mental diseases such as schizophrenia. The present data suggest that additional studies on a larger sample are needed not only to improve the statistical power regarding non-significant findings but also to compare between subtypes.

We thank Drs Berthaud, Burgat, Dubuis, and Parriaud, Hôpita du Vinatier, Lyon, France, for facilitating patient recruitment.

1 Hetrick WP, Youssef MM, Jin Y, et al. A structured clinical assessment of external sensory gating deficit in schizophreassessment of external sensory gating
nia. Schizophr Res 1995;15:177-8.

2 Pritchard WS. Cognitive event-related potential correlates of schizophrenia. Psychol Bull 1986;100:43-66.

3 Hill $\mathrm{H}$, Weisbrod $\mathrm{M}$. The relation between asymmetry and amplitude of the P300 field in schizophrenia. Clin Neurophysiol 1999;110:1611-17.

4 Faux SF, Torello MW, McCarley RW, et al. P300 in schizophrenia: confirmation and statistical validation of temporal region deficit in P300 topography. Biol Psychiatry 1988;23:776-90.

5 McCarley RW, Faux SF, Shenton ME, et al. Event-related potentials in schizophrenia: their biological and clinical cotentials in schizophrenia: their biological and clinical ogy. Schizophr Res 1991;4:209-31.

6 Adler LE, Patchman E, Franks R, et al. Neurophysiological Adler LE, Patchman E, Franks R, et al. Neurophysiological evidence for a defect in neuronal mechanisms involved in sensory

7 Boutros NN, Zouridakis G, Overall J. Replication and extension of P50 findings in schizophrenia. Clin Electroencephalogr 1991;22:40-5.

8 Ward P, Hoffer LD, Liebert BJ, et al. Replication of P50 auditory gating deficit in Australian patients with schizophrenia. Psychiatry Res 1996;64:121-35.

9 Shelley AM, Ward PB, Catts SV, et al. Mismatch negativity: an index of preattentive processing deficit in schizophrenia. Biol Psychiatry 1991;30:1059-62.

10 Javitt DC, Doneshka P, Grochowski S, et al. Impaired mismatch negativity generation reflects widespread dysfunction of working memory in schizophrenia. Arch Gen function of working mem
Psychiatry 1995;52:550-8.

11 Alain C, Hargrave R, Woods DL. Processing of auditory stimuli during visual attention in patients with schizophrenia. Biol Psychiatry 1998;44:1151-9.
12 Hirayasu Y, Potts GF, O'Donnel BF, et al. Auditory mismatch negativity in schizophrenia: evaluation with high density recording voltage. Am F Psychiatry 1998;155:12814.

13 Igata $M$, Ohta $M$, Hayashida $Y$, et al. Normalization of auditory brainstem responses resulting from improved clinical symptoms in schizophrenia. Schizophr Res 1995;16:81-2.

14 Venables P. Input dysfunction in schizophrenia. In: Maher BA, ed. Progress in experimental personality research. Orlando, FL: Academic Press, 1964:1-47.

15 Gruzelier J. The cardiac response of schizophrenics to orienting signal and non-signal tones. Biol Psychiatry 1975; 3:143-55.

16 McGhie A, Chapman J. Disorders of attention and perception in early schizophrenia. Br f Med Psychol 1961;34:10316.

17 Gruzelier J, Hammond NV. Gain, losses, and lateral differences in the hearing of unmedicated schizophrenic patients. Br F Psychol 1979;70:319-30.

18 Mathew VM, Gruzelier J, Liddle PF. Lateral asymmetries in auditory acuity distinguish hallucinating from nonhallucinating schizophrenic patients. Psychiatry Res 1992; 46:127-138.

19 Dallos P. The active cochlea. F Neurosci 1992;12:4575-85.

20 Rasmussen GL. Efferent fibers of the cochlear nerve and cochlear nucleus. In: Rasmussen GL, Windle WL, eds. Neural mechanisms of auditory and vestibular systems. Neural mechanisms of auditory and vestibular

21 Warr WB, Guinan JJJr, White JS. Organization of the efferent fibers: the lateral and medial olivocochlear systems. In: Altshuler R, Bobbin RP, Hoffman D, eds. Neurobiology of hearing: the cochlea. New York: Raven Press, 1986:333-48.

22 Warr WB. Organization of olivocochlear efferent system in mammals. In: Webster DB, Popper AN, Fay RR, eds. Mammalian auditory pathway: neuroanatomy. New York: Springer Verlag, 1992:420-48.

23 Guinan JJJr. Physiology of olivocochlear efferents. In: Fay RR, Popper AN, eds. The cochlea. New York: Springer Verlag, 1996:435-502.

24 Kemp DT. Stimulated acoustic emissions from within the human auditory system. I Acoust Soc Am 1978;64:1386-1.

25 Brownell WE, Bader CR, Bertrand D, et al. Evoked mechanical responses of isolated cochlear outer hair cells. Science 1985;277:194-6.

26 Zenner HP. Motile responses in outer hair cells. Hear Res 1986;22:83-90.

27 Kemp DT. Otoacoustic emissions, travelling waves and cochlear mechanisms. Hear Res 1986;22, 95-104.

28 Probst R, Lonsbury-Martin BL, Martin GK. A review of otoacoustic emissions. F Acoust Soc Am 1991;89:2027-67.

$29 \mathrm{Kemp}$ DT. Evidence of mechanical nonlinearity and frequency selective wave amplification in the cochlea. Archives of Otology Rhinology and Laryngology 1979;224:37Arch.

30 Collet L, Kemp DT, Veuillet E, et al. Effect of contralateral auditory stimuli on active cochlear micro-mechanical properties in human subjects. Hear Res 1990;43:251-62.

31 Veuillet E, Duclaux R, Collet L. Effect of contralateral acoustic stimulation on active cochlear micromechanical properties in human subjects: dependence on stimulus variables. F Neurophysiol 65:724-35.

32 Mountain DC. Changes in endolymphatic potential and crossed olivocochlear bundle stimulation alter cochlear mechanics. Science 1980;210:71-2.

33 Siegel JH, Kim DO. Efferent neural control of cochlear mechanics? Olivocochlear bundle stimulation affects cochmechanics? Olivocochlear bundle stimulation affects coch-

34 Puel JL, Rebillard G. Effects of contralateral sound stimulation on the distortion product $2 \mathrm{~F} 1-\mathrm{F} 2$ : evidence that the medial efferent system is involved. $f$ Acoust Soc $\mathrm{Am}$ 1990:87:1630-5.

35 Warren EH, Liberman MC. Effects of contralateral sound on auditory nerve responses: I. Contributions of cochlear efferents. Hear Res 1989;37:89-104.

36 Williams EA, Brookes GB, Prasher DK. Effects of contralateral acoustic stimulation on acoustic emissions following vestibular neurectomy. Scandinavian Audiology 1993;22:197-203.

37 Williams EA, Brookes GB, Prasher DK. Effects of olivocochlear bundle section on otoacoustic emissions in humans: efferent effects in comparison with control subjects. Acta Otolaryngol 1994;114:121-9.

38 Giraud AL, Collet L, Chéry-Croze S, et al. Evidence of a medial olivocochlear involvement in contralateral suppres-
sion of otoacoustic emissions in humans. Brain Res sion of otoacous

39 Micheyl C, Morlet T, Giraud AL, et al. Contralateral suppression of evoked otoacoustic emissions and detection of a multi-tone complex noise. Acta Otolaryngol 1995;115: $178-82$.

40 Micheyl C, Collet L. Involvement of medial olivocochlear system in the detection of tones in noise. 7 Acoust Soc Am 1996;99:1604-10.

41 Micheyl C, Perrot X, Collet L. Relationship between auditory intensity discrimination in noise and olivocochlear efferent system activity in humans. Behav Neurosci 1997;111:801-7.

42 Collet L, Veuillet E, Bene J, et al. Effects of contralateral white noise on click-evoked emissions in normal and sensorineural ears: towards an exploration of the medial olivocochlear system. Audiology 1992;31:1-7.

43 Collet L, Roge B, Descouens D, et al. An objective auditory dysfunction in infantile autism. Lancet 1993;342:923-4. 
44 Huffman RF, Henson OWJr. The descending auditory pathway and acousticomotor systems: connections with the inferior

45 Khalfa S, Collet L. Functional asymmetry of medial olivocochlear system in humans: towards a peripheral auditory lateralization. Neuroreport 1996;7:993-6.

46 Khalfa S, Veuillet E, Collet L. Influence of handedness on peripheral auditory asymmetry. Eur $\mathcal{F}$ Neurosci 1998b;10 2731-7.

47 American Psychiatric Association. Diagnostic and statistical manual of mental disorders. 4th ed. Washington, DC: American Psychiatric Press, 1994

48 Overall JR, Gorham DR. The brief psychiatric rating scale. Fournal of Operational Psychiatry 1980;11:48-64.

49 Andreasen NC. The scale for the assessment of negative symptoms ( $S A N S$ ). Iowa City, IA: The University of Iowa, symptoms
1983.

50 Andreasen NC. The scale for the assessment of positive symptoms (SAPS). Iowa City, IA: The University of Iowa, symptoms 1984.

51 Oldfield RC. The assessment and analysis of handedness. Neuropsychologia 1971;9:97-111.

52 Kemp DT, Ryan S, Bray P. A guide to the effective use of otoacoustic emissions. Ear Hear 1990;11:93-105.

53 Veuillet E, Bazin F, Collet L. Objective evidence of periph eral auditory disorders in learning-impaired children. Four nal of Audiological Medicine 1999;8:18-29.

54 Powers NL, Salvi RJ, Wang J, et al. Elevation of auditory thresholds by spontaneous cochlear oscillations. Nature 1995;375:585-7.

55 Veuillet E, Duverdy-Bertholon F, Collet L. Effect of contralateral acoustic stimulation on the growth of click-evoked otoacoustic emissions in humans. Hear Res 1996;96:128-35.

56 Khalfa S, Micheyl C, Veuillet E, et al. L. Peripheral auditory assessment using TEOAEs. Hear Res 1998;121:29-34.

57 Geschwind N, Levitsky W. Human brain: left-right asymmetries in temporal speech region. Science 1968;161:1867 .
58 Steinmetz H. Structure, function and cerebral asymmetry: In vivo morphometry of the planum temporale. Neurosci Biobehav Rev 1996;20:587-91.

59 Binder JR, Frost JA, Hammeke TA, et al. Function of the left planum temporale in auditory and linguistic processing. Brain 1996;119:1239-47.

60 Ross CA, Pearlson GD. Schizophrenia, the heteromodal association neocortex and development: potential for a neurogenic approach. TINS 1996;19:171-6.

61 Crow TJ. Schizophrenia as a failure of hemispheric dominance for language. Trends Neurosci 1997;20:339-43.

62 Flor-Henry P. Psychosis in temporal lobe epilepsy. Epilepsia 1969;10:363-95.

63 Crow TJ. Temporal lobe asymmetries as the key to the etiology of schizophrenia. Schizophr Bull 1990;16:433-43.

64 Crow TJ, Ball J. Schizophrenia as an anomaly of development of cerebral asymmetry. A postmortem study and a proposal concerning the genetic basis of the disease. Arch Gen Psychiatry 1989;46:1145-50.

65 Wexler BE, Giller ELJr, Southwick S. Cerebral laterality, symptoms and diagnosis in psychotic patients. Biol Psychiatry 1991;29:103-16.

66 Bruder GE, Rabinowicz E, Towey J, et al. Smaller right ear (left hemisphere) advantage for dichotic fused words in patients with schizophrenia. Am f Psychiatry 1985;152: $932-5$.

67 McCarley RW, Shenton M, O'Donnell BF et al. Auditory P300 abnormalities and left posterior superior temporal gyrus volume reduction in schizophrenia. Arch Gen Psychiatry 1993;50:190-7.

68 Braff DL. Information processing and attention dysfunctions in schizophrenia. Schizophr Bull 1992;19:233-59.

69 Green JF, King DJ. Cognitive functioning in schizophrenia. CNS Drugs 1996;6:382-98.

70 Gruzelier JH. Functional neuropsychophysiological asymmetry in schizophrenia: a review and reorientation. Schizophr Bull 1999;25:91-120. 